\title{
Torque and conventional spin Hall currents in two-dimensional spin-orbit coupled systems: Universal relation and hyperselection rule
}

\author{
Tsung-Wei Chen* and Guang-Yu Guo ${ }^{\dagger}$ \\ Department of Physics and Center for Theoretical Sciences, National Taiwan University, Taipei 106, Taiwan \\ (Received 4 July 2008; revised manuscript received 1 February 2009; published 3 March 2009)
}

\begin{abstract}
We investigate torque and also conventionally defined spin Hall currents in two-dimensional (2D) spin-orbit coupled systems of spin-1/2 particles within the linear-response Kubo formalism. We obtain some interesting relations between the conventional and torque spin Hall conductivities for the generic effective Hamiltonian $H_{0}=\epsilon_{k}^{0}+A(\mathbf{k}) \sigma_{x}-B(\mathbf{k}) \sigma_{y}$, where $A(\mathbf{k})=\eta_{i}^{A} k_{i}+\eta_{i j}^{A} k_{i} k_{j}+\eta_{i j l}^{A} k_{i} k_{j} k_{l}+\cdots, B(\mathbf{k})=\eta_{i}^{B} k_{i}+\eta_{i j}^{B} k_{i} k_{j}+\eta_{i j l}^{B} k_{i} k_{j} k_{l}+\cdots$, and $\eta^{\prime} \mathrm{s}$ are the specific system-dependent coefficients. Specifically, we find that in the intrinsic case, the magnitude of torque spin Hall conductivity $\sigma_{x y}^{\tau_{z}}(0)$ is always twice larger than the conventional spin Hall conductivity $\sigma_{x y}^{s_{z}}(0)$, and the two conductivities have the opposite signs, i.e., $\sigma_{x y}^{\tau_{z}}(0)=-2 \sigma_{x y}^{s_{z}}(0)$. This universal relation, therefore, suggests that in the intrinsic case, the total spin Hall conductivity $\sigma_{x y}^{z}(0)$ in the $2 \mathrm{D}$ systems is equal to the conventional spin Hall conductivity in magnitude but has the opposite sign, namely, $\sigma_{x y}^{z}(0)=\sigma_{x y}^{\tau_{z}}(0)+\sigma_{x y}^{s_{z}}(0)$ $=-\sigma_{x y}^{s_{z}}(0)$. This universal relation also holds in the presence of a uniform in-plane magnetic field. We also find that if the 2D systems are rotationally invariant, there exists a hyperangular momentum $I_{z}=\left(\mathbf{k} \times \frac{\partial \theta}{\partial \mathbf{k}}\right)_{z} s_{z}+L_{z}$ which is conserved. Furthermore, the hyperangular-momentum current $\left\langle\frac{1}{2}\left\{I_{z}, v_{x}\right\}\right\rangle$ vanishes and this leads to a hyperselection rule for the conventional spin Hall current. In particular, in the $2 \mathrm{D} k$-linear Rashba and wurtzitetype systems, $I_{z}=s_{z}+L_{z}$, and the up(down)-spin current would always be accompanied by the down(up)-orbitalangular-momentum (OAM) current. In the 2D $k$-cubic Rashba, $I_{z}=3 s_{z}+L_{z}$, and the hyperselection rule is the same as in the $k$-linear Rashba system. In the 2D $k$-linear Dresselhaus system, on the other hand, $I_{z}=-s_{z}+L_{z}$, and the up(down)-spin current would always be followed by the up(down)-OAM current.
\end{abstract}

DOI: 10.1103/PhysRevB.79.125301

PACS number(s): 71.70.Ej, 72.25.Dc, 73.63.Hs, 85.75.-d

\section{INTRODUCTION}

Spin-current generation is an important issue in the emerging spintronics. ${ }^{1-3}$ Recent proposals of the intrinsic spin Hall effect are therefore remarkable. ${ }^{4,5}$ In the spin Hall effect (SHE), a transverse spin current is generated in response to an electric field in a system with spin-orbit coupling. ${ }^{6,7}$ This effect has been considered to arise extrinsically, i.e., by impurity scattering. ${ }^{6}$ The scattering becomes spin dependent in the presence of spin-orbit coupling, and this gives rise to the SHE. In the recent proposals, in contrast, the SHE could arise intrinsically in hole-doped ( $p$-type) bulk semiconductors ${ }^{4}$ and also in electron-doped ( $n$-type) semiconductor heterostructures ${ }^{5}$ due to intrinsic spin-orbit coupling in the band structure. This intrinsic SHE would thus provide a mechanism to generate electric-driven spin current without applied magnetic fields in semiconductors, which can be more readily integrated with well-developed semiconductor electronics. Recently, the spin accumulation at the edges of semiconductor samples which is believed to be due to the SHE has been measured optically. ${ }^{8-10}$ Further, large SHE in metallic systems even at room temperature has been detected electrically. ${ }^{11-13}$

Many theoretical papers have been written addressing various issues about the intrinsic SHE. In Ref. 4, the SHE in the $p$-type GaAs semiconductor was explained as arising from the $k$-space Berry curvature in response to the applied electric field. This intrinsic SHE would lead to the possibility that the spin-orbit coupling can be used to manipulate spin chirality in semiconductors without dissipation. In Ref. 14, it was shown that the SHE in the $p$-type GaAs semiconductor is robust against the disorder based on the parity invariance of the spherical Luttinger Hamiltonian. The Berry-phaseinduced SHE was also generalized to the case of spinning particles. ${ }^{15}$ In Ref. 16, an orbital-angular-momentum (OAM) Hall current is predicted to exist in response to an electric field and is found to cancel exactly the spin Hall current in the SHE. In Ref. 17, however, ab inito relativistic bandstructure calculations show that the OAM Hall conductivity in $p$-type semiconductors is 1 order of magnitude smaller than the spin Hall conductivity, indicating no cancellation between the spin and OAM Hall effects in bulk semiconductors. The spin Hall conductivity in the two-dimensional (2D) $k$-linear Rashba system has been shown to be suppressed by weak nonmagnetic disorder. ${ }^{18}$ However, the spin Hall conductivity calculated with the consideration of the vertex correction due to the impurity scattering does not vanish, in general, and, e.g., in 2D $k$-cubic Rashba system, ${ }^{19}$ 2D $k$-cubic wurtzite system, ${ }^{14}$ and 2D $k$-cubic Dresselhaus system..$^{20}$ Very recently, the large SHE in Pt metal at room temperature ${ }^{13}$ was also theoretically investigated and was attributed to be an intrinsic one due to the band anticrossings near the Fermi level at the $L$ and $X$ symmetry points in the Brillouin zone. ${ }^{21}$

The spin precession around the effective magnetic field caused by the spin-orbit coupling leads to the fundamental problem that the conventionally defined intuitive spincurrent operator $\frac{1}{2}\left\{\mathbf{v}, s_{z}\right\}$ is not conserved. Therefore, how to properly define the spin current operator has been intensively studied in recent years. ${ }^{22-24} \mathrm{In}$ view of the spin continuity equation $^{25} \frac{\partial \mathcal{S}_{z}}{\partial t}+\nabla \cdot \mathbf{J}_{s}=\mathcal{T}_{z}$, Shi et al. ${ }^{22}$ recently provided a proper definition of conserved spin current to resolve this issue. The effective conserved spin current $\frac{d}{d t}\left(\mathbf{x} s_{z}\right)$ constructed from the spin continuity equation is composed of 
TABLE I. Some common 2D systems where the effective Hamiltonian can be described by Eq. (1). The $\Delta(\mathbf{k})$ describes the energy dispersion in the presence of spin-orbit coupling, wherein $\gamma(\phi)=\sqrt{\alpha^{2}+\beta^{2}-2 \alpha \beta \sin (2 \phi)}, \kappa(\phi)=\frac{1}{2} \sin (2 \phi)$, and $\tan ^{-1} \phi=\frac{k_{y}}{k_{x}}$. The $\sigma_{x y}^{s_{z}}(0)$ is the conventionally defined spin Hall conductivity. The pseudospin angular momentum of the $k$-cubic Rashba hole system used in the calculation of spin current is $\mathbf{S}=\frac{3}{2} \hbar \vec{\sigma}$. Superscript $(*)$ denotes that the system is not rotationally invariant.

\begin{tabular}{lccccc}
\hline \hline 2D system & $A(\mathbf{k})$ & $B(\mathbf{k})$ & $\Delta(\mathbf{k})$ & $\sigma_{x y}^{s_{z}}(0)$ & References \\
\hline Rashba & $\alpha k_{y}$ & $\alpha k_{x}$ & $\alpha k$ & $-|e| / 8 \pi$ & 28 \\
Dresselhaus ([001]) & $\beta k_{x}$ & $\beta k_{y}$ & $\beta k$ & $|e| / 8 \pi$ & 29 \\
Dresselhaus $([110]) *$ & $\rho k_{x}$ & $-\rho k_{x}$ & $\sqrt{2} \rho k \cos \phi$ & 0 & 3 \\
Rashba-Dresselhaus* & $\alpha k_{y}-\beta k_{x}$ & $\alpha k_{x}-\beta k_{y}$ & $k \gamma(\phi)$ & $-|e| / 8 \pi \operatorname{sign}\left(\alpha^{2}-\beta^{2}\right)$ & 27,30, and 31 \\
$k$-cubic Rashba (hole) & $i \alpha_{R} / 2\left(k_{-}^{3}-k_{+}^{3}\right)$ & $\alpha_{R} / 2\left(k_{-}^{3}+k_{+}^{3}\right)$ & $\alpha_{R} k^{3}$ & $-9|e| \hbar^{2} / 16 \pi^{2} m \alpha_{R}\left(1 / k_{F}^{+}-1 / k_{F}^{-}\right)$ & 32 \\
$k$-cubic Dresselhaus $*$ & $\beta_{D} k_{x} k_{y}^{2}$ & $\beta_{D} k_{y} k_{x}^{2}$ & $\beta_{D} k^{3} \kappa(\phi)$ & $|e| \hbar^{2} / 16 \pi^{2} m \beta_{D} \int d \phi\left(\csc \phi / k_{F}^{+}(\phi)-\csc \phi / k_{F}^{-}(\phi)\right)$ & 20 \\
Wurtzite type & $\left(\alpha_{o}+\beta_{o} k^{2}\right) k_{y}$ & $\left(\alpha_{o}+\beta_{o} k^{2}\right) k_{x}$ & $\alpha_{o} k+\beta_{o} k^{3}$ & $-|e| \hbar^{2} / 16 m \pi \tan ^{-1}\left(\frac{\sqrt{\alpha_{o} \beta_{o}\left(k_{F}^{+}-k_{F}^{-}\right)}}{\alpha_{o}+\beta_{o} k_{F}^{+} k_{F}^{-}}\right) / \sqrt{\alpha_{o} \beta_{o}}$ & 10 and 33 \\
\hline \hline
\end{tabular}

two terms. One term is the conventional intuitive spincurrent operator $\frac{d \mathbf{x}}{d t} s_{z}$ and the other term $\mathbf{x} \frac{d s_{z}}{d t}$ which is the so-called torque spin current comes from the spin precessional motion. Shi et al. ${ }^{22}$ considered the spin Hall coefficients for three widely studied semiconductor models, namely, 2D k-linear Rashba, 2D k-cubic Rashba, and threedimensional (3D) Luttinger models, in the clean limit, and found that the conserved spin Hall conductivities are dramatically different from the conventional spin Hall conductivities. For example, in the 2D systems, the conserved spin Hall conductivity is equal to the conventional spin Hall conductivity in size but has an opposite sign. ${ }^{22}$ In Ref. 26, the results of calculations taking into account the conserved spin current as well as impurity scattering effect for 2D $k$-linear Rashba and $k$-cubic Rashba systems are reported. Recently, we extended the conserved definition of spin-current operator and offered a proper definition of the OAM current operator. $^{27} \mathrm{We}$ also found that in 2D Dresselhaus and Rashba-Dresselhaus systems, the conserved spin Hall conductivity is equal to the conventional spin Hall conductivity in size but has an opposite sign. ${ }^{27}$

Clearly, it is important to consider the new definition of spin current ${ }^{22,26,27}$ and it is of interest to know the torque and hence conserved spin Hall coefficients in other 2D systems. In the present paper, therefore, we study the torque, conventional, and conserved spin Hall conductivities in all 2D spinorbit coupled systems described by a generic effective Hamiltonian [Eq. (1)] within the frequency-dependent Kubo linear-response theory. The generic effective Hamiltonian covers all common 2D spin-orbit coupled systems used in the literature such as $k$-linear Rasha, Dresselhaus, RashbaDresselhaus, $k$-cubic Dresselhaus, and wurtzite-type Hamiltonians (Table I). We find two interesting universal relations among the torque, conventional, and total conserved spin Hall conductivities. Furthermore, we explore possible connections between conventional spin current and orbital motion of carriers and identify the existence of a conserved hyperangular momentum $I_{z}$ in rotationally invariant $2 \mathrm{D}$ spinorbit coupled systems. The conservation of the hyperangular momentum $I_{z}$ would lead to a hyperselection rule which dictates that the up(down)-spin state in the sense of $\left(\mathbf{k} \times \frac{\partial \theta}{\partial \mathbf{k}}\right)_{z} s_{z}$ would be accompanied by the down(up)-OAM state in these systems.
The present paper is organized as follows. In Sec. II we define a generic effective Hamiltonian for 2D spin-orbit coupled systems and calculate the time evolution of Paulispin and position operators in the Heisenberg picture. In Sec. III we calculate the conventional and torque spin Hall conductivities by using frequency-dependent Kubo formulae and also present universal relations between these conductivities. In Sec. IV we report our finding that there exists a conserved hyperangular momentum $I_{z}$ in the systems with the cylindrically symmetric energy dispersion. We also demonstrate that the existence of $I_{z}$ leads to the hyperselection rule for the conventional spin Hall current. Our conclusions are given in Sec. V. Appendixes A and C of this paper outline our derivation of the spin continuity equation, a proof of conservation of $I_{z}$, and a proof of the vanishing of the $I_{z}$ current in 2D rotational invariant systems, respectively.

\section{GENERIC MODEL HAMILTONIAN}

The effective Hamiltonian for spin-1/2 particles can be expressed as a linear combination of Pauli matrices $\sigma_{x}, \sigma_{y}$, and $\sigma_{z}$. In 2D systems, we consider the following general effective Hamiltonian:

$$
H_{0}=\epsilon_{k}^{0}+A(\mathbf{k}) \sigma_{x}-B(\mathbf{k}) \sigma_{y},
$$

where $\epsilon_{k}^{0}=\hbar^{2} k^{2} / 2 m$ is the single-particle kinetic energy, and functions $A(\mathbf{k})$ and $B(\mathbf{k})$ describe the energy dispersion caused by the spin-orbit interaction. In general, $A(\mathbf{k})$ and $B(\mathbf{k})$ can be expressed as $A(\mathbf{k})=\eta_{i}^{A} k_{i}+\eta_{i j}^{A} k_{i} k_{j}+\eta_{i j l}^{A} k_{i} k_{j} k_{l}+\cdots$ and $B(\mathbf{k})=\eta_{i}^{B} k_{i}+\eta_{i j}^{B} k_{i} k_{j}+\eta_{i j l}^{B} k_{i} k_{j} k_{l}+\cdots$, where $\eta$ 's are the coefficients to be determined for each specific system. The Einstein summation convention is used. The general properties of coefficients $\eta$ 's are determined by the symmetry requirements. For instance, time-reversal invariance of spin current $\mathcal{J}=\frac{d\left(\mathbf{x s}_{z}\right)}{d t}$ requires that $A(\mathbf{k})$ and $B(\mathbf{k})$ must be an odd function of $\mathbf{k}$, i.e., $A(-\mathbf{k})=-A(\mathbf{k})$ and $B(-\mathbf{k})=-B(\mathbf{k})$. This leads to the fact that the spin-dependent part of the Hamiltonian has no spatial inversion symmetry. In Appendix A, we show that the systems described by Eq. (1) satisfy the spin continuity equation: $\frac{\partial \mathcal{S}_{z}}{\partial t}+\nabla \cdot \mathbf{J}_{s}=\mathcal{T}_{z}$. In Table I, we list the specific functions $A(\mathbf{k})$ and $B(\mathbf{k})$ for several common $2 \mathrm{D}$ systems. However, we should stress here that the following derivation is inde- 
pendent of the detailed forms of $A(\mathbf{k})$ and $B(\mathbf{k})$.

We should emphasize that Eq. (1) is an effective Hamiltonian for 2D systems valid only near the Brillouin-zone center and is not a bare Hamiltonian that describes the band structure of the whole Brillouin zone. In other words, Eq. (1) is applicable to the $2 \mathrm{D}$ semiconductor structures with the electron or hole pocket centered at the Brillouin-zone center, such as $p$-type zinc-blende semiconductors and $n$-type wurtzite nitrides, but not to the metals with a complex Fermi surface such as platinum. ${ }^{21}$ In writing the effective Hamiltonian (1), we made the assumption that the particle spin-1/2 (or the pseudospin 1/2 for $k$-cubic Rashba Hamiltonian) lies in the two-dimensional plane. For these spin-1/2 particles, we need only the two-component Bloch wave function and thus the effective Hamiltonian can be written as the linear combination of Pauli matrices. Since the particle spin lies in the plane, the spin splittings induced by bulk or structure inversion asymmetry can be described by introducing the in-plane components of the $k$-dependent effective magnetic field, in which they are $A(\mathbf{k})$ and $B(\mathbf{k})$. The periodic potential and spin-orbit coupling effect would enter the effective Hamiltonian via the $A(\mathbf{k})$ and $B(\mathbf{k})$. The explicit forms of $A(\mathbf{k})$ and $B(\mathbf{k})$ depend on the symmetries of the underlying crystalline structure and band structure near the Brillouin-zone center. Since the 2D system we considered is time-reversal invariant (zero magnetic field), the spin splitting would result from the spatial inversion asymmetry (or structure inversion asymmetry). This implies that $A(\mathbf{k})$ and $B(\mathbf{k})$ are odd functions of $k$.

For the convenience of derivation, it turns out to be useful to introduce a vector $\vec{M}=\left(M_{x}, M_{y}\right)$. The in-plane components of vector $\vec{M}=\left(M_{x}, M_{y}\right)$ are $M_{x} \equiv \cos \theta=\frac{B}{\Delta}$ and $M_{y} \equiv \sin \theta$ $=\frac{A}{\Delta}$. The Hamiltonian [Eq. (1)] can now be rewritten as

$$
H_{0}=\epsilon_{k}^{0}+\Delta(\vec{\sigma} \times \vec{M})_{z},
$$

where $\Delta(\mathbf{k})=\left(A^{2}+B^{2}\right)^{1 / 2}$ is the energy dispersion of spin splitting determined by the explicit forms of $A(\mathbf{k})$ and $B(\mathbf{k})$ (e.g., Table I). The vector Pauli matrix used in Eq. (2) is $\vec{\sigma}$ $=\left(\sigma_{x}, \sigma_{y}\right)$. The eigenenergy of Eq. (2) is $E_{n}(\mathbf{k})=\epsilon_{k}^{0}-n \Delta(\mathbf{k})$ and the corresponding eigenvector is given by

$$
|n \mathbf{k}\rangle=\frac{1}{\sqrt{2}}\left(\begin{array}{c}
e^{-i \theta(\mathbf{k})} \\
\text { in }
\end{array}\right),
$$

where the $\theta(\mathbf{k})$ is

$$
\theta(\mathbf{k})=\tan ^{-1}\left(\frac{A(\mathbf{k})}{B(\mathbf{k})}\right)
$$

and the band index is denoted as $n= \pm$. It is straightforward to show that $(\vec{\sigma} \times \vec{M})_{z}^{2}=\left(M_{x}^{2}+M_{y}^{2}\right)=1$. The time evolution operator $\exp \left(i H_{0} t / \hbar\right)$ can be further written as

$$
e^{i H_{0} t / \hbar}=e^{i \epsilon_{k}^{0} t / \hbar}\left[\cos \left(\frac{\Omega t}{2}\right)+i(\vec{\sigma} \times \vec{M})_{z} \sin \left(\frac{\Omega t}{2}\right)\right],
$$

where $\Omega=2 \Delta / \hbar$. By using the definition of Heisenberg picture for Schrödinger operator

$$
\mathcal{O}, \mathcal{O}(t)=\exp \left(i H_{0} t / \hbar\right) \mathcal{O} \exp \left(-i H_{0} t / \hbar\right),
$$

one can show that the time evolution of Pauli-spin operators are given by

$$
\begin{gathered}
\sigma_{x}(t)=\sigma_{x}-M_{x} \sin (\Omega t) \sigma_{z}+M_{x}(\vec{\sigma} \cdot \vec{M})[\cos (\Omega t)-1], \\
\sigma_{y}(t)=\sigma_{x}-M_{y} \sin (\Omega t) \sigma_{z}+M_{y}(\vec{\sigma} \cdot \vec{M})[\cos (\Omega t)-1], \\
\sigma_{z}(t)=\cos (\Omega t) \sigma_{z}+(\vec{\sigma} \cdot \vec{M}) \sin (\Omega t) .
\end{gathered}
$$

It can be shown that $\langle n \mathbf{k}|(\vec{\sigma} \cdot \vec{M})| n \mathbf{k}\rangle=\left\langle n \mathbf{k}\left|\sigma_{z}\right| n \mathbf{k}\right\rangle=0$ by the use of the eigenstate in Eq. (3). This means that the expectation value of the $z$ component of the spin operator vanishes in the absence of electric field. The time evolution position operator can be written as $\mathbf{x}(t)=\mathbf{x}(0)+\delta \mathbf{x}(t)$ and

$$
\begin{aligned}
\delta \mathbf{x}(t)= & {\left[\frac{\partial \epsilon_{k}^{0}}{\hbar \partial \mathbf{k}}+\frac{1}{2}(\vec{\sigma} \times \vec{M})_{z}\left(\frac{\partial \Omega}{\partial \mathbf{k}}\right)\right] t+\frac{1}{2} \frac{\partial \theta}{\partial \mathbf{k}} } \\
& \times\left\{[\cos (\Omega t)-1] \sigma_{z}+(\vec{\sigma} \cdot \vec{M}) \sin (\Omega t)\right\},
\end{aligned}
$$

where $\mathbf{x}(0)$ is the initial condition and $\delta \mathbf{x}(t=0)=0$. It can be shown that in the pure Rashba system, Eq. (7) would reproduce the result given in Ref. 34. The physical meaning of each term is as follows. If the spin-orbit coupling vanishes, one has $\Delta=0$, and the time evolution position operator (7) reduces to the free particle equation of motion $\mathbf{x}(t)=\mathbf{x}(0)$ $+\frac{\partial \epsilon_{k}^{0}}{\hbar \delta \mathbf{k}} t$. The second term of Eq. (7) is the displacement arising from the anomalous velocity in the presence of spin-orbit coupling. The anomalous velocity plays an important role in the anomalous Hall effect. ${ }^{35}$ The third and fourth terms have the oscillation behavior inducing the Zitterbewegung. ${ }^{34,36,37}$

\section{SPIN HALL CONDUCTIVITY}

As mentioned before, the conserved spin current is divided into two terms,

$$
\frac{d}{d t}\left(\mathbf{x} s_{z}\right)=\frac{d \mathbf{x}}{d t} s_{z}+\mathbf{x} \frac{d s_{z}}{d t} .
$$

In addition to the conventional spin current $\frac{d \mathbf{x}}{d t} s_{z}$, one has to introduce the torque spin current $\mathbf{x} \frac{d s_{z}}{d t}$ in order to satisfy the conserved spin continuity equation. On the other hand, the time-reversal symmetry of the conserved spin current (8) would lead to the spatial inversion asymmetry of the spindependent part of Hamiltonian (1). This can be seen as follows. From the commutator $\frac{1}{i \hbar}\left[\sigma_{z}, H_{0}\right]=\Omega \vec{\sigma} \cdot \vec{M}$, since the position operator is even under time-reversal operation, the invariance of torque spin current under time-reversal symmetry must require $A(-\mathbf{k})=-A(\mathbf{k})$ and $B(-\mathbf{k})=-B(\mathbf{k})$. The time evolution of the conserved spin current is

$$
\begin{aligned}
\mathcal{J}(t) & =\frac{1}{2}\left\{\mathbf{v}(t), s_{z}(t)\right\}+\frac{1}{2}\left\{\mathbf{x}(t), \frac{1}{i \hbar}\left[s_{z}, H_{0}\right](t)\right\} \\
& \equiv \mathbf{J}^{s_{z}}(t)+\mathbf{J}_{z}^{\tau_{z}}(t)+\mathbf{J}^{\tau_{0}}(t),
\end{aligned}
$$

where 


$$
\mathbf{J}^{s_{z}}(t)=\frac{1}{2}\left\{\mathbf{v}(t), s_{z}(t)\right\}
$$

is the conventional spin current,

$$
\mathbf{J}^{\tau_{z}}(t)=\frac{1}{2}\left\{\delta \mathbf{x}(t), \frac{1}{i \hbar}\left[s_{z}, H_{0}\right](t)\right\}
$$

is the torque spin current which is independent of the choice of origin of coordinate system and

$$
\mathbf{J}^{\tau_{0}}(t)=\frac{1}{2}\left\{\mathbf{x}(0), \frac{1}{i \hbar}\left[s_{z}, H_{0}\right](t)\right\}
$$

is the other part of torque spin current which depends on the initial choice of the origin of the coordinate system. The time-dependent part of the position operator $\delta \mathbf{x}(t)$ is given by Eq. (7). It can be shown that $\left\langle n \mathbf{k}\left|\mathbf{J}^{s_{z}}(t=0)\right| n \mathbf{k}\right\rangle=\langle n \mathbf{k}| \mathbf{J}^{\tau_{z}}(t$ $=0)|n \mathbf{k}\rangle=0$ and $\left\langle n \mathbf{k}\left|\mathbf{J}^{\tau_{0}}(t=0)\right| n \mathbf{k}\right\rangle=0$. This leads to the fact that the conserved spin current vanishes at $t=0$ as required, namely, $\langle n \mathbf{k}|\mathcal{J}(t=0)| n \mathbf{k}\rangle=0$ in the absence of external electric field. The value of Eq. (12) is the torque spin current with reference to the initial choice of the origin of the coordinate system. We could choose the initial position of the carrier as the origin of the coordinate system and, as a result, Eq. (12) would not contribute to the spin accumulation. In that sense, the conserved spin current $\mathcal{J}(t)$ can be divided into two terms $\mathcal{J}(t)=\tilde{\mathcal{J}}(t)+\mathbf{J}^{\tau_{0}}$, where

$$
\tilde{\mathcal{J}}(t)=\mathbf{J}^{s_{z}}(t)+\mathbf{J}^{\tau_{z}}(t)
$$

corresponds to the total spin current which is free from the choice of the origin of the coordinate system. Equation (13) could satisfy the initial condition, namely, $\langle n \mathbf{k}|\widetilde{\mathcal{J}}(t=0)| n \mathbf{k}\rangle$ $=0$ because it can be shown that $\left\langle n \mathbf{k}\left|\mathbf{J}^{s_{z}}(t=0)\right| n \mathbf{k}\right\rangle=0$ and $\left\langle n \mathbf{k}\left|\mathbf{J}_{z}(t=0)\right| n \mathbf{k}\right\rangle=0$. The frequency-dependent Kubo formula for a spatially homogeneous electric field ${ }^{38}$ is

$$
\begin{aligned}
\sigma_{\mu \nu}(\omega)= & \frac{q / \hbar}{(\omega+i \eta)} \int_{0}^{\infty} d t e^{i(\omega+i \eta) t} \frac{1}{V} \sum_{n \mathbf{k}} f_{n \mathbf{k}} \\
& \times\left\langle n \mathbf{k}\left|\left[J_{\mu}(t), v_{\nu}(0)\right]\right| n \mathbf{k}\right\rangle,
\end{aligned}
$$

where $q$ is the carrier charge, i.e., $q=-|e|$ for electrons, and $f_{n \mathbf{k}}$ is the Fermi distribution at zero temperature. The parameter $\eta$ is used to regularize the integral and the direction of external electric field is denoted as index $\nu$. We will calculate the conventional and torque spin Hall conductivities by using Eq. (14). We assume that the electric field is applied in the $y$ direction $(\nu \rightarrow y)$. The transverse spin current is composed of conventional and torque spin Hall currents $\tilde{\mathcal{J}}_{x}(t)=J_{x}^{s_{z}}(t)$ $+J_{x}^{\tau_{z}}(t)$. The conventional spin Hall current in the $x$ direction is $J_{x}^{s_{z}}(t)=\frac{1}{2}\left\{v_{x}, s_{z}\right\}(t)$, and it can be evaluated as

$$
J_{x}^{s_{z}}(t)=\frac{\hbar}{2} \widetilde{v}_{x} \sigma_{z}(t)
$$

where $\widetilde{v}_{x}$ is defined as $\widetilde{v}_{x}=\hbar k_{x} / m$. The torque spin Hall current in the $x$ direction is $J_{x}^{\tau_{z}}(t)=\frac{\hbar}{2} \frac{1}{2}\left\{\delta x(t), \frac{1}{i \hbar}\left[\sigma_{z}, H_{0}\right]\right\}$. After substitution of the commutator $\frac{1}{i \hbar}\left[\sigma_{z}, H_{0}\right]=\Omega \vec{\sigma} \cdot \vec{M}$ to the torque current and straightforward calculation, one can obtain

$$
J_{x}^{\tau_{z}}(t)=\frac{\hbar}{2}\left[\left(\widetilde{v}_{x} t\right) \Omega \vec{\sigma}(t) \cdot \vec{M}+\frac{\Omega}{2} \frac{\partial \theta}{\partial k_{x}} \sin (\Omega t)\right],
$$

where $\delta x(t)$ given in Eq. (7) was used and $\vec{\sigma}(t)$ $=\left(\sigma_{x}(t), \sigma_{y}(t)\right)$ wherein $\sigma_{x}(t)$ and $\sigma_{y}(t)$ are given in Eq. (6). With the definition of conserved spin current, the total spin Hall conductivity is the sum of contributions of conventional and torque spin Hall currents,

$$
\sigma_{x y}^{z}(\widetilde{\omega})=\sigma_{x y}^{s_{z}}(\widetilde{\omega})+\sigma_{x y}^{\tau_{z}}(\widetilde{\omega}),
$$

where $\widetilde{\omega}=\omega+i \eta$. The first and second terms in the right-hand side of equality correspond to the conventional spin Hall current and spin torque current, respectively. By using Eq. (14) and $v_{y}(0)=\widetilde{v}_{y}+\frac{\partial \Delta}{\hbar \partial k_{y}}(\vec{\sigma} \times \vec{M})_{z}+\frac{\partial \theta}{\hbar \partial k_{y}} \Delta(\vec{\sigma} \cdot \vec{M})$, one can obtain

$$
\sigma_{x y}^{s_{z}}(\widetilde{\omega})=\frac{-q}{4 \pi^{2} m} \int_{k_{F}^{-}}^{k_{F}^{+}} d S_{k} \frac{\Delta\left(k_{x} \frac{\partial \theta}{\partial k_{y}}\right)}{\widetilde{\omega}^{2}-\Omega^{2}},
$$

for the conventional spin Hall conductivity and

$$
\sigma_{x y}^{\tau_{x}}(\widetilde{\omega})=\frac{-2 q}{\pi^{2} m \hbar^{2}} \int_{k_{F}^{-}}^{k_{F}^{+}} d S_{k} \frac{\Delta^{3}\left(k_{x} \frac{\partial \theta}{\partial k_{y}}\right)}{\left[\widetilde{\omega}^{2}-\Omega^{2}\right]^{2}},
$$

for torque spin Hall conductivity, where $\widetilde{\omega}=\omega+i \eta, \Omega$ $=2 \Delta / \hbar, k_{F}^{ \pm}$is Fermi momentum for band $n= \pm$, and $d S_{k}$ $=k d k d \phi$. In the absence of spin-orbit coupling, $\Delta=0$, the spin Hall conductivity vanishes as one can see from Eqs. (18) and (19).

\section{A. Static limit: $\widetilde{\omega}=\mathbf{0}$}

In the intrinsic case and the static limit (i.e., $\widetilde{\omega}=0$ ), after some algebraic calculations, one can obtain from Eqs. (18) and (19) an exact relation

$$
\sigma_{x y}^{\tau_{z}}(0)=-2 \sigma_{x y}^{s_{z}}(0) .
$$

Thus, the torque spin Hall conductivity is simply a constant (-2) multiple of the conventional spin Hall conductivity. This universal relation implies that in the spin-1/2 2D systems with spin-orbit coupling, the magnitude of the torque spin Hall conductivity is always twice larger than the conventional spin Hall conductivity and has an opposite sign. The spin $z$ component is not a constant of motion, as shown by the commutator of the $s_{z}$ and $H_{0}$. We find that, by virtue of the commutation properties of Pauli-spin-1/2 matrices, $\left[s_{z}, H_{0}\right]$ is a linear combination of the in-plane spin components, namely, $x$ and $y$ components. We can rewrite the Hamiltonian as $H_{0}=\epsilon_{k}^{0}+\vec{\sigma} \cdot \overrightarrow{\mathcal{B}}_{\text {eff }}$, where $\overrightarrow{\mathcal{B}}_{\text {eff }}$ is the effective magnetic field in the $\mathbf{k}$ space. The time derivative of spin $z$ component is $d \sigma_{z} / d t=(-2)\left(\vec{\sigma} \times \overrightarrow{\mathcal{B}}_{\text {eff }}\right)_{z} / \hbar$ and the quantity -2 on the right-hand side of equality actually yields the result that the magnitude of intrinsic torque spin Hall conductivity is always twice larger than conventional spin Hall conductivity. Physically, it is the spin precession that leads to the result that the magnitude of torque spin Hall conductivity is 
twice larger than conventional, and the sign of torque spin Hall conductivity is opposite to the conventional spin Hall conductivity. We notice that the $k$-cubic Rashba system describing spin-3/2 heavy hole also obeys Eq. (20). In the $k$-cubic Rashba system, ${ }^{32}$ Pauli matrices operate on the states with spin-3/2 projection along the growth direction. In that sense, the $k$-cubic Rashba system actually represents a pseudospin-1/2 system. We also note that the spin-dependent part of the Hamiltonian of the $k$-cubic Rashba is originally written as $\frac{i \alpha_{R}}{2}\left(k_{-}^{3} \sigma_{+}-k_{+}^{3} \sigma_{-}\right)$, where $k_{ \pm}=k_{x} \pm i k_{y}$ and $\sigma_{ \pm}$ $=\sigma_{x} \pm i \sigma_{y}$. This can be rewritten as $A(\mathbf{k}) \sigma_{x}-B(\mathbf{k}) \sigma_{y}$ with $A$ $=\frac{i \alpha_{R}}{2}\left(k_{-}^{3}-k_{+}^{3}\right)$ and $B=\frac{\alpha_{R}}{2}\left(k_{-}^{3}+k_{+}^{3}\right)$. The simple relation between intrinsic conventional and torque spin Hall conductivities (20) is independent of the detailed forms of spin splitting [i.e., $A(\mathbf{k})$ and $B(\mathbf{k})$ ] and hence the energy dispersion [i.e., $\Delta(\mathbf{k})]$. The total intrinsic spin Hall conductivity $\sigma_{x y}^{z}(0)$ $=\left[\sigma_{x y}^{s_{z}}(0)+\sigma_{x y}^{\tau_{z}}(0)\right]$ is then given by

$$
\sigma_{x y}^{z}(0)=-\sigma_{x y}^{s_{z}}(0) \text {. }
$$

The sign of the total spin Hall conductivity is always opposite to the sign of the conventional spin Hall conductivity. Even if higher order $k$ terms were included in the theoretical calculations, the conclusions described above would still be true. It should be emphasized that the validity of Eq. (21) is independent of the sign of carrier charge. Interestingly, Eq. (21) suggests that the properties of the total intrinsic spin Hall conductivity can be characterized by the conventional spin Hall conductivity only. Both Eqs. (20) and (21) are the universal results for 2D spin-orbit coupled systems. Let us now apply formulae (18) and (19) to some specific systems. In the Rashba-Dresselhaus system, for example, we can derive from Eqs. (18) and (19) that $\sigma_{x y}^{s_{z}}=(q / 8 \pi) \operatorname{sign}\left(\alpha^{2}-\beta^{2}\right)$ and $\sigma_{x y}^{\tau_{z}}=-(q / 4 \pi) \operatorname{sign}\left(\alpha^{2}-\beta^{2}\right)$. The results agree with previous works for the Rashba system ${ }^{22}$ and for the RashbaDresselhaus system. ${ }^{27}$ We note that when $\alpha=\beta, A=-B$ and $\theta$ is independent of $\mathbf{k}$. In this case, the spin Hall conductivity vanishes. ${ }^{27,31}$ For the Dresselhaus Hamiltonian along the [110] direction (see Table I), we have $A=-B$ and thus the spin Hall conductivity also vanishes. It has been shown that the [110] Dresselhaus Hamiltonian with $\hat{e}_{z}$ along [001] direction is different from the Rashba-Dresselhaus model with $\alpha$ $=\beta$ only by a unitary transformation. ${ }^{39}$ The exact $\mathrm{SU}(2)$ spin rotation symmetry has been investigated in both systems. ${ }^{39}$

We also note that Eq. (21) is still true even if there exists a uniform in-plane external magnetic field $\overrightarrow{\mathcal{B}}$, as can be seen as follows. The interaction of the spin and magnetic field is proportional to $\vec{\sigma} \cdot \overrightarrow{\mathcal{B}}=\sigma_{x} \mathcal{B}_{x}-\sigma_{y}\left(-\mathcal{B}_{y}\right)$. The Hamiltonian is now given by $H=\epsilon_{k}^{0}+\sigma_{x}\left[A(k)+\mu_{B} \mathcal{B}_{x}\right]-\sigma_{y}\left[B(\mathbf{k})-\mu_{B} \mathcal{B}_{y}\right]$, where $\mu_{B}$ is the Bohr magneton. We can redefine functions $A^{\prime}(\mathbf{k})$ and $B^{\prime}(\mathbf{k})$ as $A^{\prime}(\mathbf{k})=A(k)+\mu \mathcal{B}_{x}$ and $B^{\prime}(\mathbf{k})=B(\mathbf{k})$ $-\mu \mathcal{B}_{y}$, respectively. Therefore, though the numerical values of Eqs. (18) and (19) may change, Eq. (20) is still valid in the presence of a uniform in-plane magnetic field.

Our predictions of, e.g., the conserved spin Hall current and conductivity [Eq. (21)], can be tested by direct measurements of the spin Hall current or conductivity. In particular, our prediction that the total spin Hall conductivity differs from the conventional spin Hall conductivity only in sign
[Eq. (21)] could be easily tested. As for the induction of a magnetic field by a charge current, a spin current would generate an electric field. ${ }^{23,40}$ Therefore, our prediction for the conserved spin Hall current could be tested by a comparison of the measured spin-current-induced electric field to the numerical simulation for, e.g., a mesoscopic spin-orbit coupled system. Another kind of experiment is to determine the inverse spin Hall conductivity by measuring the charge current and Zeeman field gradient. ${ }^{22}$ The sign and magnitude of the conserved spin Hall conductivity can then be obtained via the Onsager relation. ${ }^{22}$ Indeed, large spin Hall effect in metallic systems at room temperature has recently been detected by the method of inverse spin Hall effect. ${ }^{11-13}$ Hopefully, our interesting predictions would stimulate measurements of inverse spin Hall effect in 2D semiconductor systems in the near future.

\section{B. Finite frequency case: $\widetilde{\boldsymbol{\omega}} \neq \mathbf{0}$}

At finite frequencies, the torque spin Hall conductivity is not a constant multiple of conventional spin Hall conductivity. Nonetheless, we find that Eq. (18) is related to Eq. (19) by the following equation:

$$
\sigma_{x y}^{\tau_{z}}(\widetilde{\omega})=\left(-2-\widetilde{\omega} \frac{\partial}{\partial \widetilde{\omega}}\right) \sigma_{x y}^{s_{z}}(\widetilde{\omega}) .
$$

The second term in Eq. (22) comes from the variation of the k-space effective magnetic field with the frequencydependent external electric field. Taking into account the torque spin current, we find that the total spin Hall conductivity is directly related to the conventional spin Hall conductivity still. Substituting Eq. (22) into Eq. (17), we obtain the relationship between conventional spin Hall conductivity $\sigma_{x y}^{s_{z}}(\widetilde{\omega})$ and total spin Hall conductivity $\sigma_{x y}^{z}(\widetilde{\omega})$ in the presence of nonzero frequency-dependent electric field,

$$
\sigma_{x y}^{z}(\widetilde{\omega})=-\frac{\partial}{\partial \widetilde{\omega}}\left[\widetilde{\omega} \sigma_{x y}^{s}(\widetilde{\omega})\right] .
$$

Equation (23) shows that the total spin Hall conductivity can be determined directly from the frequency spectrum of conventional spin Hall conductivity. Unlike the static limit, the total ac spin Hall conductivity is not proportional to the conventional spin Hall conductivity. It follows from Eq. (23) that the step-function behavior of the conventional spin Hall conductivity would result in a large response of the total spin Hall conductivity. This large response has recently been investigated in the Rashba-Dresselhaus system in Ref. 30. It can be shown that Eqs. (18) and (19) for the RashbaDresselhaus system agree with the results in Ref. 30. We also find that the simple relation between the torque and conventional spin Hall conductivities [Eq. (22)] would be maintained even when the external magnetic field is applied.

\section{CONSTANT OF MOTION IN ROTATIONALLY INVARIANT SYSTEMS}

\section{A. Constant of motion}

If a $2 \mathrm{D}$ system is invariant under rotation about the $z$ axis, its energy dispersion is cylindrically symmetric, i.e., $\Delta$ 
$=\Sigma_{\ell} c_{\ell} k^{\ell}(\ell=1,2,3, \ldots)$. We find that in this case, there exists a conserved quantity whose operator $I_{z}$ is defined as

$$
I_{z}=\left(\mathbf{k} \times \frac{\partial \theta}{\partial \mathbf{k}}\right)_{z} s_{z}+L_{z} .
$$

Let us call this quantity $I_{z}$ the hyperangular momentum. We can show that $I_{z}$ satisfies the following commutation relation (see Appendix B):

$$
\left[I_{z}, H_{0}\right]=0,
$$

where $s_{z}=\frac{\hbar}{2} \sigma_{z}, L_{z}=\hbar\left(x k_{y}-y k_{x}\right)$ is the $z$ component of the $\mathrm{OAM}$, and $H_{0}$ is given in Eq. (1) or Eq. (2). Interestingly, this implies that in the rotationally invariant spin-orbit coupled systems, the flow of $\left(\mathbf{k} \times \frac{\partial \theta}{\partial \mathbf{k}}\right)_{z} s_{z}$ would be accompanied by the orbital-angular momentum $L_{z}$, and the combination of these quantities is actually a constant of motion. As a result, the spin current would in general be accompanied by the OAM current because of the spin-orbit coupling. In that sense, the external electric field would induce the current of angular momentum $\left(\mathbf{k} \times \frac{\partial \theta}{\partial \mathbf{k}}\right)_{z} s_{z}$ and the OAM current simultaneously.

Further, it can be shown that the hyperangular-momentum Hall current $\left\langle\frac{1}{2}\left\{I_{z}, v_{x}\right\}\right\rangle$ vanishes in the steady-state case within the linear-response Kubo formalism (see Appendix C). It follows that the up-spin (down-spin) state in the sense of $\left(\mathbf{k} \times \frac{\partial \theta}{\partial \mathbf{k}}\right)_{z} s_{z}$ would be accompanied by the down-OAM (upOAM) state, rendering the hyperangular momentum conserved. Let us now apply this result to some specific systems. For the wurtzite-type and $k$-linear Rashba systems, $I_{z}=s_{z}$ $+L_{z}$, i.e., the hyperangular momentum is equal to the total angular momentum. For the $k$-cubic Rashba hole system, one would have $I_{z}=3 s_{z}+L_{z}$. Therefore, Eq. (24) gives the correct pseudospin angular momentum of hole which is $\frac{3}{2} \hbar \sigma_{z}$. The conservation of hyperangular momentum in these systems would then lead to the result that the spatial trajectory of a down-spin $\left(-\hat{e}_{z}\right)$ carrier would behave as having its orbitalangular momentum pointed to $+\hat{e}_{z}$ and vice versa [see Fig. 1(a)]. In other words, in these systems, the situation of an up-spin (down-spin) state accompanied by an up-OAM (down-OAM) trajectory is forbidden. This is a hyperselection rule that is present in the cylindrically symmetric $2 \mathrm{D}$ spin-orbit coupled systems. It must be emphasized that this hyperselection rule depends on the quantity $\left(\mathbf{k} \times \frac{\partial \theta}{\partial \mathbf{k}}\right)_{z}$. For example, in the $k$-linear Dresselhaus system, we find that $I_{z}$ $=-s_{z}+L_{z}$. Therefore, in contrast to the $k$-linear and wurtzitetype systems, the hyperselection rule in the $k$-linear Dresselhaus system implies that an up-spin (down-spin) state would be accompanied by an up-OAM (down-OAM) state [see Fig. 1(b)]. The situation of an up-spin state accompanied by an down-OAM is now forbidden in this Dresselhaus system. Nevertheless, we should emphasize that the electric fieldinduced OAM current would not result in magnetization accumulation at the edges of sample. This is due to the fact that the OAM is not an intrinsic quantity of electrons or holes, i.e., the magnetic moment associated with the orbital-angular momentum would vanish when the carrier velocity reaches zero at the edges of sample, as can be understood from the definition of $L_{z}$. Therefore, the magnetic-moment accumula- a)

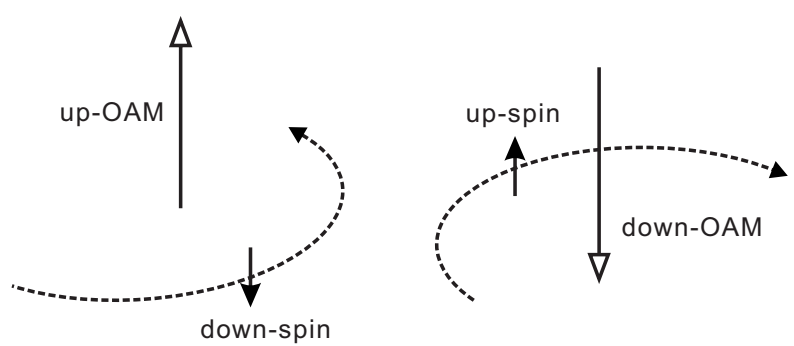

b)

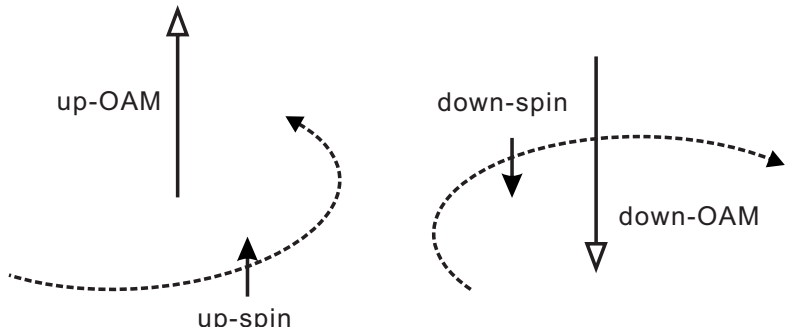

FIG. 1. Schematic diagrams showing the relationship between spin and OAM of a carrier. The direction of the OAM is determined by the right-handed sense. The dotted line illustrates the spatial trajectory of a carrier. (a) for the Wurtzite-type, $k$-linear, and $k$-cubic Rashba systems and (b) for the $k$-linear Dresselhaus system.

tion at the edges of sample would come from the spin angular momentum only. In short, it is interesting to notice that the topological quantity $\left(\mathbf{k} \times \frac{\partial \theta}{\partial \mathbf{k}}\right)_{z}$ is an integer number for $k$-linear Rashba, $k$-linear Dresselhaus, $k$-cubic Rashba, and wurtzite-type system; whereas they are $1,-1,3$, and 1 , respectively. The topological number indicates that the constant of motion in 2D rotationally invariant system is the hyperangular momentum rather than simply the total angular momentum $s_{z}+L_{z}$.

It should be pointed out that although both hyperangularmomentum conservation and hyperselection rule exist in rotationally invariant systems, this rotational symmetry may be broken when higher-order terms of $k_{i}$ are included in the $A(\mathbf{k})$ and $B(\mathbf{k})$ in Eq. (1). It would be necessary to include the higher-order terms of $k_{i}$ in Eq. (1) when the 2D semiconductor systems considered have a very large carrier concentration. When the weak symmetry-breaking higher-order terms do appear, the hyperangular momentum [Eq. (24)] is no longer conserved but the universal relation [Eq. (20)] still holds.

In Sec. IV B, we will describe the close relation between Berry vector potential and hyperangular momentum.

\section{B. Geometrical interpretation}

As mentioned above, the quantity $\left(\mathbf{k} \times \frac{\partial \theta}{\partial \mathbf{k}}\right)_{z} s_{z}$ together with the orbital-angular momentum is conserved in a rotationally invariant system. In the following, let us explain that the quantity $\left(\mathbf{k} \times \frac{\partial \theta}{\partial \mathbf{k}}\right)_{z}$ actually comes from the topological properties of the Berry vector potential. In the $2 \mathrm{D}$ systems with a cylindrically symmetric dispersion, the vector $\frac{\partial \theta}{\partial \mathbf{k}}$, in general, is perpendicular to the wave vector $\mathbf{k}$, i.e., $\mathbf{k} \cdot \frac{\partial \theta}{\partial \mathbf{k}}=0$. For example, it can be shown that the wurtzite-type, $k$-linear 


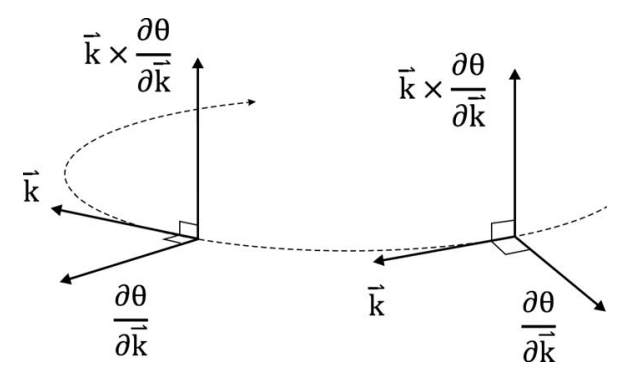

FIG. 2. A schematic diagram showing the relationship between vectors $\mathbf{k}, \frac{\partial \theta}{\partial \mathbf{k}}$, and $\mathbf{k} \times \frac{\partial \theta}{\partial \mathbf{k}}$.

Rashba, $k$-cubic Rashba, and $k$-linear Dresselhaus systems and the dot product of $\mathbf{k}$ and $\frac{\partial \theta}{\partial \mathbf{k}}$ are zero. In that sense, the three vectors $\mathbf{k}, \frac{\partial \theta}{\partial \mathbf{k}}$, and $\mathbf{k} \times \frac{\partial \theta}{\partial \mathbf{k}}$ form an orthogonal frame fixed on the carrier (see Fig. 2).

On the other hand, the Berry vector potential in a system described by Hamiltonian Eq. (2) can be written as

$$
\mathcal{A}(\mathbf{k})=\left\langle n \mathbf{k}\left|i \frac{\partial}{\partial \mathbf{k}}\right| n \mathbf{k}\right\rangle=\frac{1}{2} \frac{\partial \theta}{\partial \mathbf{k}},
$$

where the eigenvector (3) was used. The projection of spin operator onto the in-plane axes contains two terms. One term is the so-called spin helicity $\vec{\sigma} \cdot \mathbf{k}$ in the 2D spin-orbit coupled system and the other is the projection of spin on the Berry vector potential $\mathcal{A} \cdot \vec{\sigma}$. The quantity $\left(\mathbf{k} \times \frac{\partial \theta}{\partial \mathbf{k}}\right)_{z} s_{z}$ then comes from the noncommutativeness of the two in-plane projections, viz.,

$$
[\mathbf{k} \cdot \vec{\sigma}, \mathcal{A} \cdot \vec{\sigma}]=\left[\mathbf{k} \cdot \vec{\sigma}, \frac{1}{2} \frac{\partial \theta}{\partial \mathbf{k}} \cdot \vec{\sigma}\right]=i\left(\mathbf{k} \times \frac{\partial \theta}{\partial \mathbf{k}}\right)_{z} \sigma_{z}
$$

where the commutation relations of Pauli matrices were used. The overall coefficient of $\left(\mathbf{k} \times \frac{\partial \theta}{\partial \mathbf{k}}\right)_{z} s_{z}$ cannot be determined by the commutation relation alone. However, in the system with the cylindrically symmetric dispersion, the hyperangular-momentum conservation forces the overall coefficient of $\left(\mathbf{k} \times \frac{\partial \theta}{\partial \mathbf{k}}\right)_{z} s_{z}$ to be unity. Similar to the topological force induced by the noncommutative position operator, ${ }^{4}$ the spin part of conservation of the hyperangular momentum comes from the noncommutative properties of $\vec{\sigma} \cdot \mathcal{A}$ and $\vec{\sigma} \cdot \mathbf{k}$, whereas they are the projection of spin on the two orthogonal axes. Finally, it must be stressed that in the systems with the noncylindrically symmetric dispersion, the quantity (k $\left.\times \frac{\partial \theta}{\partial \mathbf{k}}\right)_{z} s_{z}$ can also be defined as the noncommutativeness of spin helicity $\vec{\sigma} \cdot \mathbf{k}$ and $\vec{\sigma} \cdot \mathcal{A}$. However, in that case, the quantity $\left(\mathbf{k} \times \frac{\partial \theta}{\partial \mathbf{k}}\right)_{z} s_{z}$ plus the orbital-angular momentum are not conserved. Therefore, the orbital motion of carrier does not accompany with $\left(\mathbf{k} \times \frac{\partial \theta}{\partial \mathbf{k}}\right)_{z} s_{z}$; namely, the hyperangular angular momentum is not conserved in this case.

The conserved quantity in spin-orbit coupled systems can be written as the sum of the spin and orbital terms. In the free-atomic case, the spin term is just the Pauli-spin operator. When the crystal environment is included, the spin term appears to be different from the Pauli-spin operator. It has a nontrivial dependence on energy dispersion that arises from the spin-orbit coupled effect. We find that the general coef- ficient is a nontrivial multiplication of $\left(\mathbf{k} \times \frac{\partial \theta}{\partial \mathbf{k}}\right)_{z}$ in rotationally invariant system. The quantity $\left(\mathbf{k} \times \frac{\partial \theta}{\partial \mathbf{k}}\right)_{z}$ is proportional to the expectation value of the orbital-angular momentum.

It is of course not surprising that a conserved angular momentum would exist in rotationally invariant systems. The conserved angular momentum in spin-orbit coupled systems would be the sum of the spin and orbital terms. In the freeatom case, the spin term is just the Pauli-spin operator, and the sum of the spin and orbital terms is indeed the total angular momentum. However, we find here that in the presence of the crystal environment, the spin term is not necessarily equal to the Pauli-spin operator but has a nontrivial dependence on the energy dispersion [Eq. (24)] that arises from the spin-orbit coupling effect. Therefore, we would like to use the hyperangular momentum here to differentiate Eq. (24) from the well-known expression of the total angular momentum in the free-atom case. Furthermore, in the systems described by the generic Hamiltonian [Eq. (1)], we find that the coefficient in the spin term in Eq. (24) is related to the Berry vector potential of the underlying band structure.

\section{CONCLUSIONS}

In conclusion, we have derived some interesting relations between the conventional and torque spin Hall conductivities for all 2D spin-orbit coupled systems described by the generic effective Hamiltonian (1) in the presence of frequencydependent electric field. In particular, we find that a universal relation $[$ Eq. (21)] between total and conventional spin Hall conductivities, i.e., $\sigma_{x y}^{z}(0)=-\sigma_{x y}^{s_{z}}(0)$ for $\tilde{\omega}=0$. Equation $(21)$ is independent of the detailed form of energy dispersion $\Delta(\mathbf{k})$ [i.e., $A(\mathbf{k})$ and $B(\mathbf{k})]$, and hence its validity is not restricted to the systems listed in Table I. We also found that in 2D rotationally invariant systems, a conserved hyperangular momentum $I_{z}$ exists and the hyperangular momentum current vanishes. This would result in a hyperselection rule that the up-spin (down-spin) state in the sense of $\left(\mathbf{k} \times \frac{\partial \theta}{\partial \mathbf{k}}\right)_{z} s_{z}$ would be accompanied by the down-OAM (up-OAM) state. Finally, we explained that the spin-dependent part of $I_{z}$ comes from the noncommutative property of spin helicity $\vec{\sigma} \cdot \mathbf{k}$ and $\vec{\sigma} \cdot \mathcal{A}$.

\section{ACKNOWLEDGMENTS}

The authors would like to thank S.-Q. Shen and M. C. Chang for useful discussions. The authors gratefully acknowledge financial support from the National Science Council and NCTS of Taiwan.

\section{APPENDIX A: SPIN CONTINUITY EQUATION}

In this appendix, we show that the Hamiltonian

$$
H=\frac{\mathbf{p}^{2}}{2 m}+H_{\mathrm{so}}+V(\mathbf{x})
$$

would satisfy the spin continuity equation,

$$
\frac{\partial \mathcal{S}_{z}}{\partial t}+\nabla \cdot \mathbf{J}^{s}=\mathcal{T}_{z}
$$

where $H_{\mathrm{so}}=A(\mathbf{p}) \sigma_{x}-B(\mathbf{p}) \sigma_{y}$ describes the spin-orbit interaction, $V(\mathbf{x})=-q \mathbf{E} \cdot \mathbf{x}$ is the potential induced by a homoge- 
neous electric field $\mathbf{E}, \mathcal{S}_{z}=\Psi^{\dagger} s_{z} \Psi$ is the spin density, $\mathbf{J}^{s}$ $=\operatorname{Re}\left[\Psi^{\dagger} \frac{1}{2}\left\{\mathbf{v}, s_{z}\right\} \Psi\right]$ is the conventional spin current, and $\mathcal{T}_{z}$ $=\operatorname{Re}\left[\Psi^{\dagger} \frac{1}{i \hbar}\left(s_{z}, H_{0}\right) \Psi\right]$ is the source term of spin current. The carrier velocity is defined as $\mathbf{v}=\frac{\partial H}{\partial \mathbf{p}}$. The real part (imaginary part) of $[\cdots]$ is denoted as $\operatorname{Re}[\cdots](\operatorname{Im}[\cdots])$ and $s_{z}=\frac{\hbar}{2} \sigma_{z}$. By using the Schrodinger equation with the two-component wave function $\Psi=\left(\begin{array}{c}\phi_{\uparrow} \\ \phi_{\downarrow}\end{array}\right)$,

$$
i \hbar \frac{\partial \Psi}{\partial t}=H \Psi
$$

one can obtain

$$
\begin{aligned}
i \hbar \frac{\partial}{\partial t}\left(\Psi^{\dagger} s_{z} \Psi\right)= & {\left.\left[-\left(\frac{\mathbf{p}^{2}}{2 m} \Psi\right)\right)^{\dagger} s_{z} \Psi+\Psi^{\dagger} s_{z}\left(\frac{\mathbf{p}^{2}}{2 m} \Psi\right)\right] } \\
& +\left[-\left(H_{\mathrm{so}} \Psi\right)^{\dagger} s_{z} \Psi+\Psi^{\dagger} s_{z} H_{\mathrm{so}} \Psi\right] \\
& +\left\{-[V(\mathbf{x}) \Psi]^{\dagger} s_{z} \Psi+\Psi^{\dagger} s_{z} V(\mathbf{x}) \Psi\right\} .
\end{aligned}
$$

We note that $\left\{\left[H_{\mathrm{so}}+V(\mathbf{x})\right] \Psi\right\}^{\dagger} s_{z} \Psi=\left\{\Psi^{\dagger} s_{z}\left[H_{\mathrm{so}}+V(\mathbf{x})\right] \Psi\right\}^{\dagger}$ because the Pauli-spin matrix $\sigma_{z}$ satisfies $\sigma_{z}^{\dagger}=\sigma_{z}$, i.e., is Hermitian. On the other hand, one can also show that

$$
\left[\Psi^{\dagger} s_{z} \mathbf{p}^{2} \Psi-\left(\mathbf{p}^{2} \Psi\right)^{\dagger} s_{z} \Psi\right]=2 \mathbf{p} \cdot \operatorname{Re}\left[\Psi^{\dagger} \mathbf{p} s_{z} \Psi\right] .
$$

After substituting Eq. (A5) into Eq. (A4), one gets

$$
\begin{aligned}
i \hbar \frac{\partial}{\partial t}\left(\Psi^{\dagger} s_{z} \Psi\right)= & \mathbf{p} \cdot \operatorname{Re}\left[\Psi^{\dagger} \frac{\mathbf{p}}{m} s_{z} \Psi\right]+2 i \operatorname{Im}\left[\Psi^{\dagger} s_{z} H_{\mathrm{so}} \Psi\right] \\
& +2 i \operatorname{Im}\left[\Psi^{\dagger} s_{z} V(\mathbf{x}) \Psi\right] .
\end{aligned}
$$

We also note that $s_{z} H_{\text {so }}$ can be written as $s_{z} H_{\text {so }}=\frac{1}{2}\left\{s_{z}, H_{\text {so }}\right\}$ $+\frac{1}{2}\left[s_{z}, H_{\text {so }}\right]$, where the first term vanishes because the Pauli matrices satisfy $\left\{\sigma_{i}, \sigma_{j}\right\}=2 \delta_{i j}$. One can obtain

$$
\begin{aligned}
\operatorname{Im}\left[\Psi^{\dagger} s_{z} H_{\text {so }} \Psi\right] & =\operatorname{Im}\left[\Psi^{\dagger} \frac{1}{2}\left[s_{z}, H_{\text {so }}\right] \Psi\right] \\
& =\frac{\hbar}{2} \operatorname{Re}\left[\Psi^{\dagger} \frac{1}{i \hbar}\left[s_{z}, H_{\text {so }}\right] \Psi\right] .
\end{aligned}
$$

The last term of Eq. (A6) vanishes because $V(\mathbf{x})$ is real as required by the Hermitian property of Hamiltonian (A1). Finally, substitution of Eq. (A7) into Eq. (A6) yields

$$
\begin{aligned}
\frac{\partial}{\partial t}\left(\Psi^{\dagger} s_{z} \Psi\right)= & -\nabla \cdot \operatorname{Re}\left[\Psi^{\dagger} \frac{1}{2}\left\{\mathbf{v}, s_{z}\right\} \Psi\right] \\
& +\operatorname{Re}\left[\Psi^{\dagger} \frac{1}{i \hbar}\left[s_{z}, H_{\mathrm{so}}\right] \Psi\right],
\end{aligned}
$$

where the commutation relation $\left\{\frac{\partial H_{\mathrm{so}}}{\partial \mathbf{p}}, s_{z}\right\}=0$ was used. Equation (A8) is the desired spin continuity equation. The average spin torque vanishes, ${ }^{22}$ and hence we have $\int d \mathbf{x} \mathcal{T}_{z}=0$. The spin torque density can be written as the divergence of spin torque dipole density $\mathbf{P}_{\tau}(\mathbf{x})$, namely, $\mathcal{T}_{z}=-\nabla \cdot \mathbf{P}_{\tau}(\mathbf{x})$. On the other hand, the spin dipole density vanishes outside the sample, and we have $\int_{V} d \mathbf{x} \mathbf{P}_{\tau}=\int_{V} d \mathbf{x}\left(-\mathbf{x} \nabla \cdot \mathbf{P}_{\tau}\right)=\int_{V} d \mathbf{x}\left(\mathbf{x} \mathcal{T}_{z}\right)$. Therefore, the spin dipole density can be written as $\mathbf{P}_{\tau}(\mathbf{x})$
$=\operatorname{Re}\left[\Psi^{\dagger} \frac{1}{2}\left\{\mathbf{x}, \frac{d s_{z}}{d t}\right\} \Psi\right]$. Finally, the effective conserved spin continuity equation can be written as

$$
\frac{\partial \mathcal{S}_{z}}{\partial t}+\nabla \cdot \mathcal{J}(\mathbf{x})=0
$$

where $\mathcal{J}(\mathbf{x})=\operatorname{Re}\left[\Psi^{\dagger} \hat{\mathcal{J}} \Psi\right]$ and the effective conserved spincurrent operator is $\hat{\mathcal{J}}=\frac{1}{2}\left\{\mathbf{v}, s_{z}\right\}+\frac{1}{2}\left\{\mathbf{x}, \frac{d s_{z}}{d t}\right\}$ which is the sum of conventional and torque spin currents.

\section{APPENDIX B: CONSERVATION OF $\boldsymbol{I}_{z}$}

In this appendix, we demonstrate that the hyperangular momentum $I_{z}$ defined in Eq. (24) is a conserved quantity when the energy dispersion $\Delta$ is rotationally invariant. First, it can be shown that the velocity operator can be written as

$$
v_{x}=\frac{1}{i \hbar}\left[x, H_{0}\right]=\widetilde{v}_{x}+\frac{\partial \Delta}{\hbar \partial k_{x}}(\vec{\sigma} \times \vec{M})_{z}+\frac{\partial \theta}{\hbar \partial k_{x}} \Delta(\vec{\sigma} \cdot \vec{M}),
$$

where $H_{0}=\epsilon_{k}^{0}+\Delta(\vec{\sigma} \times \vec{M})_{z}$ was used. The $y$ component of velocity can be obtained by replacing the index $x$ by $y$. The $z$ component of orbital-angular momentum operator is defined as $L_{z}=\hbar\left(x k_{y}-y k_{x}\right)$. Using the velocity operator, the commutator $\left[L_{z}, H_{0}\right]$ is straightforwardly evaluated as follows:

$$
\begin{aligned}
\frac{1}{i \hbar}\left[L_{z}, H_{0}\right]= & \hbar\left(v_{x} k_{y}-v_{y} k_{x}\right)=\Delta\left(\frac{\partial \theta}{\partial k_{x}} k_{y}-\frac{\partial \theta}{\partial k_{y}} k_{x}\right)(\vec{\sigma} \cdot \vec{M}) \\
& +\left(\frac{\partial \Delta}{\partial k_{x}} k_{y}-\frac{\partial \Delta}{\partial k_{y}} k_{x}\right)(\vec{\sigma} \times \vec{M})_{z} .
\end{aligned}
$$

We now define the operator $I_{z}$ as

$$
I_{z}=\left(\mathbf{k} \times \frac{\partial \theta}{\partial \mathbf{k}}\right) s_{z}+L_{z}
$$

where $\theta=\tan ^{-1}\left(\frac{A}{B}\right)$ and $s_{z}=\frac{\hbar}{2} \sigma_{z}$ is the Pauli-spin operator. Using Eqs. (B2) and (B3), we obtain

$$
\frac{1}{i \hbar}\left[I_{z}, H_{0}\right]=\left(\frac{\partial \Delta}{\partial k_{x}} k_{y}-\frac{\partial \Delta}{\partial k_{y}} k_{x}\right)(\vec{\sigma} \times \vec{M})_{z},
$$

where $\frac{1}{i \hbar}\left[\sigma_{z}, H_{0}\right]=\frac{2 \Delta}{\hbar} \vec{\sigma} \cdot \vec{M}$ was used. Equation (B4) is the main result of this appendix, and it means that, in general, the $I_{z}$ operator is not a conserved quantity. The right-hand side of Eq. (B4) explicitly depends on the form of energy dispersion. It is interesting to note that the spin term of hyperangular momentum [Eq. (B3)] is not the Pauli matrices with the multiplication of $\hbar / 2$ but the multiplication of

$$
\left(\mathbf{k} \times \frac{\partial \theta}{\partial \mathbf{k}}\right)_{z}
$$

which is further explained in Sec. IV B.

Now consider a 2D system with the cylindrically symmetric energy dispersion that can be written as the power series of magnitude of $\mathbf{k}$ denoted as $k=|\mathbf{k}|$, namely, $\Delta=\Sigma_{\ell} c_{\ell} k^{\ell}$. The right-hand side of equality in Eq. (B4) then becomes 


$$
\left(\frac{\partial \Delta}{\partial k_{x}} k_{y}-\frac{\partial \Delta}{\partial k_{y}} k_{x}\right)=\sum_{\ell} c_{\ell} \ell k^{\ell-1}\left(\frac{k_{x}}{k} k_{y}-\frac{k_{y}}{k} k_{x}\right)=0 .
$$

Therefore, the hyperangular momentum $I_{z}$ is a conserved quantity in the rotationally invariant $2 \mathrm{D}$ systems.

\section{APPENDIX C: NULL HYPERANGULAR MOMENTUM CURRENT}

In this appendix, we will show that the hyperangular momentum current $\frac{1}{2}\left\{I_{z}, \mathbf{v}\right\}$ vanishes in the linear-response regime. In the static case, the Kubo formula can be written as

$$
\begin{aligned}
\sigma_{\mu \nu}= & q \hbar \sum_{n \neq n^{\prime}} \sum_{\mathbf{k}} \frac{f_{n \mathbf{k}}-f_{n^{\prime} \mathbf{k}}}{\left[E_{n}(\mathbf{k})-E_{n^{\prime}}(\mathbf{k})\right]^{2}} \operatorname{Im}\left\langle n \mathbf{k}\left|J_{\mu}\right| n^{\prime} \mathbf{k}\right\rangle \\
& \times\left\langle n^{\prime} \mathbf{k}\left|v_{\nu}\right| n \mathbf{k}\right\rangle,
\end{aligned}
$$

where both eigenstate $|n \mathbf{k}\rangle$ and eigenenergy $E_{n}(\mathbf{k})$ are given in Sec II. In the following, the external electric field is assumed to be applied in the $y$ direction, and we calculate the conductivity $\sigma_{x y}$. First of all, the hyperangular momentum current can be divided into two terms $J_{x}^{I_{z}}=J_{x}^{\mathcal{S}_{z}}+J_{x}^{L_{z}}$. First term is the hyperspin current

$$
J_{x}^{\mathcal{S}_{z}}=\frac{1}{2}\left\{\left(\mathbf{k} \times \frac{\partial \theta}{\partial \mathbf{k}}\right)_{z} s_{z}, v_{x}\right\},
$$

corresponding to the hyperspin Hall conductivity $\sigma_{x y}^{\mathcal{S}_{z}}$. The second term of $J_{z}^{I_{z}}$ is the orbital current

$$
J_{x}^{L_{z}}=\frac{1}{2}\left\{L_{z}, v_{x}\right\}
$$

corresponding to the orbital-Hall conductivity $\sigma_{x y}^{L_{z}}$. As a result, the hyperangular momentum Hall conductivity can be written as

$$
\sigma_{x y}^{I_{z}}=\sigma_{x y}^{\mathcal{S}_{z}}+\sigma_{x y}^{L_{z}} .
$$

We first calculate the orbital-Hall conductivity. By using the velocity operator (B1), we have

$$
\begin{aligned}
\operatorname{Im}\left\langle n \mathbf{k}\left|J_{x}^{L_{z}}\right|-n \mathbf{k}\right\rangle\left\langle-n \mathbf{k}\left|v_{y}\right| n \mathbf{k}\right\rangle \\
=n \frac{\hbar k_{x}}{2 m} \Delta \frac{\partial \theta}{\partial k_{y}}\left(\frac{\partial \theta}{\partial \mathbf{k}} \times \mathbf{k}\right)_{z}-\frac{1}{2 \hbar} \operatorname{Im}\left\{i \Delta \frac { \partial \theta } { \partial k _ { y } } \left(i \frac{\partial A}{\partial k_{x}}\right.\right. \\
\left.\left.+\frac{\partial B}{\partial k_{x}}\right) e^{-i \theta}\right\}\left(\frac{\partial \theta}{\partial \mathbf{k}} \times \mathbf{k}\right)_{z}
\end{aligned}
$$

The second term of Eq. (C5) does not contribute to the orbital-Hall conductivity $\sigma_{x y}^{L_{z}}$ because of the even power of band index $n$. The orbital-Hall conductivity $\sigma_{x y}^{L_{z}}$ with the substitution of Eq. (C5) gives

$$
\sigma_{x y}^{L_{z}}=\frac{q \hbar^{2}}{16 \pi^{2} m} \int_{k_{F}^{-}}^{k_{F}^{+}} d S_{k} \frac{k_{x} \frac{\partial \theta}{\partial k_{y}}}{\Delta}\left(\frac{\partial \theta}{\partial \mathbf{k}} \times \mathbf{k}\right)_{z} .
$$

We now consider the hyperspin Hall current. Taking into account the hyperspin and the velocity operator (B1), we have

$$
\operatorname{Im}\left\langle n \mathbf{k}\left|J_{x}^{\mathcal{S}_{z}}\right|-n \mathbf{k}\right\rangle\left\langle-n \mathbf{k}\left|v_{y}\right| n \mathbf{k}\right\rangle=n \frac{\hbar k_{x}}{2 m} \Delta \frac{\partial \theta}{\partial k_{y}}\left(\mathbf{k} \times \frac{\partial \theta}{\partial \mathbf{k}}\right)_{z}
$$

Inserting Eq. (C7) into $\sigma_{x y}^{\mathcal{S}_{z}}$, we obtain

$$
\sigma_{x y}^{\mathcal{S}_{z}}=\frac{q \hbar^{2}}{16 \pi^{2} m} \int_{k_{F}^{-}}^{k_{F}^{+}} d S_{k} \frac{k_{x} \frac{\partial \theta}{\Delta k_{y}}}{\Delta}\left(\mathbf{k} \times \frac{\partial \theta}{\partial \mathbf{k}}\right)_{z} .
$$

Comparison of Eqs. (C6) and (C8) gives $\sigma_{x y}^{\mathcal{S}_{z}}=-\sigma_{x y}^{L_{z}}$. As a result, we have $\sigma_{x y}^{I_{z}}=\sigma_{x y}^{\mathcal{S}_{z}}+\sigma_{x y}^{L_{z}}=0$, i.e., the hyperangular momentum current is zero. *twchen@phys.ntu.edu.tw

†gyguo@phys.ntu.edu.tw

${ }^{1}$ G. A. Prinz, Science 282, 1660 (1998).

${ }^{2}$ S. A. Wolf, D. D. Awschalom, R. A. Buhrman, J. M. Daughton, S. von Molnar, M. L. Roukes, A. Y. Chtchelkanova, and D. M. Treger, Science 294, 1488 (2001).

${ }^{3}$ I. Žutić, J. Fabian, and D. Sarma, Rev. Mod. Phys. 76, 323 (2004).

${ }^{4}$ S. Murakami, N. Nagaosa, and S.-C. Zhang, Science 301, 1348 (2003); Phys. Rev. B 69, 235206 (2004).

${ }^{5}$ J. Sinova, D. Culcer, Q. Niu, N. A. Sinitsyn, T. Jungwirth, and A. H. MacDonald, Phys. Rev. Lett. 92, 126603 (2004).

${ }^{6}$ M. I. Dyakonov and V. I. Perel, Phys. Lett. 35, 459 (1971).

${ }^{7}$ J. E. Hirsch, Phys. Rev. Lett. 83, 1834 (1999).

${ }^{8}$ K. Kato, R. C. Myers, A. C. Gossard, and D. D. Awschalom, Science 306, 1910 (2004).

${ }^{9}$ J. Wunderlich, B. Kaestner, J. Sinova, and T. Jungwirth, Phys. Rev. Lett. 94, 047204 (2005).

${ }^{10}$ H. J. Chang, T.-W. Chen, J. W. Chen, W. C. Hong, W. C. Tsai, Y.
F. Chen, and G. Y. Guo, Phys. Rev. Lett. 98, 136403 (2007).

${ }^{11}$ E. Saitoh, M. Ueda, H. Miyajima, and G. Tatara, Appl. Phys. Lett. 88, 182509 (2006).

${ }^{12}$ S. O. Valenzuela and M. Tinkham, Nature (London) 442, 176 (2006).

${ }^{13}$ T. Kimura, Y. Otani, T. Sato, S. Takahashi, and S. Maekawa, Phys. Rev. Lett. 98, 156601 (2007); 98, 249901(E) (2007).

${ }^{14}$ S. Murakami, Phys. Rev. B 69, 241202(R) (2004).

${ }^{15}$ A. Bérard and H. Mohrbach, Phys. Lett. A 352, 190 (2006).

${ }^{16}$ S. Zhang and Z. Yang, Phys. Rev. Lett. 94, 066602 (2005).

${ }^{17}$ G. Y. Guo, Y. Yao, and Q. Niu, Phys. Rev. Lett. 94, 226601 (2005).

${ }^{18}$ J. I. Inoue, G. E. W. Bauer, and L. W. Molenkamp, Phys. Rev. B 70, 041303(R) (2004); R. Raimondi and P. Schwab, Phys. Rev. B 71, 033311 (2005); O. Chalaev and D. Loss, Phys. Rev. B 71, 245318 (2005); V. V. Bryksin and P. Kleinert, ibid. 73, 165313 (2006); K. Arii, M. Koshino, and T. Ando, ibid. 76, 045311 (2007); B. Zhou, C. X. Liu, and S. Q. Shen, Europhys. Lett. 79, 47010 (2007); J. Sinova, S. Murakami, S.-Q. Shen, and M.-S. 
Choi, Solid State Commun. 138, 214 (2006).

${ }^{19}$ B. A. Bernevig and S. C. Zhang, Phys. Rev. Lett. 95, 016801 (2005).

${ }^{20}$ A. G. Mal'shukov and K. A. Chao, Phys. Rev. B 71, 121308(R) (2005).

${ }^{21}$ G. Y. Guo, S. Murakami, T.-W. Chen, and N. Nagaosa, Phys. Rev. Lett. 100, 096401 (2008).

${ }^{22}$ J. Shi, P. Zhang, D. Xiao, and Q. Niu, Phys. Rev. Lett. 96, 076604 (2006).

${ }^{23}$ Q.-F. Sun and X. C. Xie, Phys. Rev. B 72, 245305 (2005).

${ }^{24}$ R. Shen, Y. Chen, Z. D. Wang, and D. Y. Xing, Phys. Rev. B 74, 125313 (2006); Y. Wang, K. Xia, Z.-B. Su, and Z. Ma, Phys. Rev. Lett. 96, 066601 (2006); P.-Q. Jin, Y.-Q. Li, and F.-C. Zhang, J. Phys. A 39, 7115 (2006); H.-T. Yang and C. Liu, Phys. Rev. B 75, 085314 (2007); A. Vernes, B. L. Györffy, and P. Weinberger, ibid. 76, 012408 (2007); F. J. Huang, R. Qi, Y. D. Li, and W. M. Liu, Europhys. Lett. 79, 10004 (2007).

${ }^{25}$ D. Culcer, J. Sinova, N. A. Sinitsyn, T. Jungwirth, A. H. MacDonald, and Q. Niu, Phys. Rev. Lett. 93, 046602 (2004).

${ }^{26}$ N. Sugimoto, S. Onoda, S. Murakami, and N. Nagaosa, Phys. Rev. B 73, 113305 (2006).

${ }^{27}$ T. W. Chen, C. M. Huang, and G. Y. Guo, Phys. Rev. B 73, 235309 (2006).

${ }^{28}$ Y. A. Bychkov and E. I. Rashba, J. Phys. C 17, 6039 (1984); J. Nitta, T. Akazaki, H. Takayanagi, and T. Enoki, Phys. Rev. Lett. 78, 1335 (1997).

${ }^{29}$ G. Dresselhaus, Phys. Rev. 100, 580 (1955).
${ }^{30}$ A. Wong, J. A. Maytorena, C. López-Bastidas, and F. Mireles, Phys. Rev. B 77, 035304 (2008).

${ }^{31}$ S. Q. Shen, Phys. Rev. B 70, 081311(R) (2004); M. C. Chang, ibid. 71, 085315 (2005).

${ }^{32}$ J. Schliemann and D. Loss, Phys. Rev. B 71, 085308 (2005); R. Winkler, ibid. 62, 4245 (2000); R. Winkler, H. Noh, E. Tutuc, and M. Shayegan, ibid. 65, 155303 (2002); M. G. Pala, M. Governale, J. Konig, U. Zulicke, and G. Iannaccone, ibid. 69, 045304 (2004).

${ }^{33}$ I. Zorkani and E. Kartheuser, Phys. Rev. B 53, 1871 (1996); V. I. Litvinov, ibid. 76, 245305 (2007).

${ }^{34}$ J. Schliemann, D. Loss, and R. M. Westervelt, Phys. Rev. Lett. 94, 206801 (2005); Phys. Rev. B 73, 085323 (2006).

${ }^{35}$ M. C. Chang and Q. Niu, Phys. Rev. Lett. 75, 1348 (1995); Phys. Rev. B 53, 7010 (1996); G. Sundaram and Q. Niu, ibid. 59, 14915 (1999); A. Crépieux and P. Bruno, ibid. 64, 014416 (2001); V. K. Dugaev, P. Bruno, M. Taillefumier, B. Canals, and C. Lacroix, ibid. 71, 224423 (2005).

${ }^{36}$ J. Cserti and G. Dávid, Phys. Rev. B 74, 172305 (2006).

${ }^{37}$ E. Bernardes, J. Schliemann, M. Lee, J. C. Egues, and D. Loss, Phys. Rev. Lett. 99, 076603 (2007).

${ }^{38}$ J. Schliemann and D. Loss, Phys. Rev. B 69, 165315 (2004).

${ }^{39}$ B. A. Bernevig, J. Orenstein, and S.-C. Zhang, Phys. Rev. Lett. 97, 236601 (2006).

${ }^{40}$ Q.-F. Sun, H. Guo, and J. Wang, Phys. Rev. B 69, 054409 (2004). 